\title{
Prediction error and trace dominance determine the fate of fear memories after post-training manipulations
}

\author{
Joaquín M. Alfei, ${ }^{1,4}$ Roque I. Ferrer Monti, ${ }^{1,4}$ Victor A. Molina, ${ }^{2}$ Adrián M. Bueno, ${ }^{1}$ \\ and Gonzalo P. Urcelay ${ }^{3}$ \\ ${ }^{1}$ Laboratorio de Psicología Experimental, Facultad de Psicología; ${ }^{2}$ Departamento de Farmacología, Facultad de Ciencias Químicas, \\ Universidad Nacional de Córdoba, Córdoba, 5000, Argentina; ${ }^{3}$ Department of Psychology and Behavioural and Clinical Neuroscience \\ Institute, University of Cambridge, Cambridge CB2 3EB, United Kingdom
}

\begin{abstract}
Different mnemonic outcomes have been observed when associative memories are reactivated by CS exposure and followed by amnestics. These outcomes include mere retrieval, destabilization-reconsolidation, a transitional period (which is insensitive to amnestics), and extinction learning. However, little is known about the interaction between initial learning conditions and these outcomes during a reinforced or nonreinforced reactivation. Here we systematically combined temporally specific memories with different reactivation parameters to observe whether these four outcomes are determined by the conditions established during training. First, we validated two training regimens with different temporal expectations about US arrival. Then, using Midazolam (MDZ) as an amnestic agent, fear memories in both learning conditions were submitted to retraining either under identical or different parameters to the original training. Destabilization (i.e., susceptibly to MDZ) occurred when reactivation was reinforced, provided the occurrence of a temporal prediction error about US arrival. In subsequent experiments, both treatments were systematically reactivated by nonreinforced context exposure of different lengths, which allowed to explore the interaction between training and reactivation lengths. These results suggest that temporal prediction error and trace dominance determine the extent to which reactivation produces the different outcomes.
\end{abstract}

[Supplemental material is available for this article.]

Memory storage was traditionally conceived as a fixed and static process (McGaugh 1966). However, a large body of evidence in experimental psychology suggested malleability for previously stored memories through different manipulations (e.g., Misanin et al. 1968), and this issue has recently regained interest (Nader et al. 2000; Nader 2013). Data from a variety of experimental preparations has firmly established that consolidated memories become vulnerable to amnestics (i.e., sensitive to different manipulations), if reactivated under appropriate conditions (Piñeyro et al. 2013). ${ }^{5}$ Understanding the dynamics of memory reactivation and extinction has strong theoretical and clinical value, offering avenues to advance translatable knowledge on how human and nonhuman animals use stored information and pointing to potential therapeutic targets for the treatment of pathogenic memories (Urcelay 2012).

Memory reactivation was conceptualized by Lewis (1979) as a process that reactivates a stored, inactive memory into an active state that can encourage appropriate behavioral responses. Under some conditions, reactivation procedures lead to memory destabilization, rendering the mnemonic trace vulnerable to the

${ }^{5}$ Following this reasoning, diverse pharmacological and behavioral treatments applied in the reactivation session could theoretically rewrite the previously stable representation. Alternatively, it has widely been proposed that new memories are presumably being formed during the reminder session and they potentially compete with the initial memory for retrieval and/or expression (Miller and Matzel 2000, 2006).

${ }^{4}$ These authors contributed equally to this work.

Corresponding author: gonurcelay@gmail.com

Article is online at http://www.learnmem.org/cgi/doi/10.1101//m.038513.115. effects of amnestic treatments. However, reactivation, retrieval, destabilization, and reconsolidation are not identical constructs (Guisquet-Verrier and Riccio 2012). In the context of this study, memory reactivation is used as a generic term which refers to the process through which a memory in a latent (inactive) state is placed in an active state by the reexposure to the conditioned stimulus. It is from this active state that memory can be retrieved leading to a behavioral output.

Because reactivation does not always lead to destabilizationreconsolidation, studies on human and nonhuman animals have been concerned with the specific conditions that lead to destabilization of a consolidated memory. One candidate mechanism widely developed in theories of learning (Bush and Mosteller 1955; Rescorla and Wagner 1972; Sutton and Barto 1981) that has been suggested to be critical for memory destabilization is prediction error (Pedreira et al. 2004). The psychological intuition is that a discrepancy between what is expected (based on information stored in long-term memory) and what is experienced (during reactivation) triggers the destabilization process to update the memory, which then requires reconsolidation in order to stabilize the newly incorporated information (Pedreira et al. 2004). This implies that memory reactivation will lead to memory destabilization if, and only if, a prediction error is experienced by the animal. This hypothesis has recently found support both in

(C) 2015 Alfei et al. This article is distributed exclusively by Cold Spring Harbor Laboratory Press for the first 12 months after the full-issue publication date (see http://learnmem.cshlp.org/site/misc/terms.xhtml). After 12 months, it is available under a Creative Commons License (Attribution-NonCommercial 4.0 International), as described at http://creativecommons.org/licenses/ by-nc/4.0/ 
humans and rats through two manipulations that induce an error prediction during memory reactivation: (a) varying the amount of CS or response exposure (Exton-McGuinness et al. 2014b, 2015; Reichelt et al. 2012, 2013; Sevenster et al. 2012, 2013, 2014), and (b) varying the temporal expectation of the US presentation (Diaz-Mataix et al. 2013).

In the case of associative memories, an often used reactivation protocol to induce memory destabilization involves the presentation of a previously conditioned stimulus (CS) in the absence of the unconditioned stimulus (US), which is by definition a standard procedure to induce extinction learning (Pavlov 1927; Bouton 2004). In other words, memory reactivation without US presentation can lead to different mnemonic outcomes: mere retrieval, destabilization-reconsolidation or extinction. When reviewing the literature to better understand these seemingly contradictory outcomes, a pattern is seen: for example, Pedreira and Maldonado (2003) (also see Eisenberg et al. 2003) observed that reactivation duration influences in part which process takes place. This pattern has been replicated by other groups that further found that short presentations only produce memory retrieval, intermediate reactivations destabilize the memory and long reactivations generate extinction learning (Suzuki et al. 2004; Bustos et al. 2009; Piñeyro et al. 2013).

It is well established that extinction learning is best captured as new CS-NoUS learning (Bouton 1993) and thus many researchers have assessed what effect, if any, do amnestics have after extended post-training exposure to a CS. The results suggest that with enough CS exposure the new (extinction) memory is dominant, and administration of an amnestic will have the effect of disrupting the extinction memory, leading to an increase in responding at test relative to the appropriate control conditions (Eisenberg et al. 2003; Lee et al. 2006). Intriguingly, recent studies have shown that intermediate amounts of posttraining CS exposure, a point at which presumably neither CS-US nor CS-NoUS trace is dominant, have revealed insensitivity to amnestic (Flavell and Lee 2013; Merlo et al. 2014; Sevenster et al. 2014).

We hypothesized that the occurrence of these four possible outcomes from a memory reactivation, mere retrieval, destabilization-reconsolidation, transitional insensitive period, and extinction learning depends upon the combination of both conditions, the initial learning experience and the CS-reactivation length. We decided to address this hypothesis experimentally, using contextual fear conditioning (CFC) and rats as experimental subjects.

In Experiment 1 we varied the amount of context exposure immediately before shock presentation ( 1 or $5 \mathrm{~min}$ ) in order to establish two single-event learning experiences with different expectations about US arrival. A minute-by-minute analysis of behavior during a nonreinforced test $3 \mathrm{~d}$ later revealed clear temporal control of the conditioned response (CR, freezing), for it peaked and decreased according to the time point at which the US was delivered during training. This suggests the existence of different expectations about US arrival as a function of training parameters (also see Savastano and Miller 1998). Then, by varying the temporal expectation of the US presentation during reinforced reactivations (CS-US) and using MDZ as an amnestic agent, we replicated the aforementioned results of Díaz-Mataix et al. (2013), showing that US temporal prediction error is critical for memory destabilization-reconsolidation (Exps 2 and 3). In subsequent experiments (Exps 4-8), by a systematic combination of both temporal-specific memories with different CS-reactivation lengths, we demonstrated that the necessary time to reveal these four possible outcomes (mere retrieval, destabilization-reconsolidation, transitional insensitive period, and extinction) from a post-training CS presentation is highly dependent on the conditions established during training.
It is important to mention that throughout these experiments the two learning conditions ( 1 and 5 min of context exposure in training) were run separately, except in Experiment 1 which included both conditions thereby providing some justification for the subsequent between-experiment comparisons. To avoid confounds that can arise from cross-experiment comparisons, the corresponding statistical analyses were performed separately within each experiment. However, the experiments are presented in pairs, according to the reactivation duration, since this would best highlight how different learning conditions produce different mnemonic processes with equivalent amounts of reactivation.

\section{Results}

\section{Experiment 1}

Animals are capable of learning when the US arrives based on CS duration (Savastano and Miller 1998; Balsam et al. 2001; Gallistel and Balsam 2014). Indeed, temporally specific CRs have been observed in fear-potentiated startle after a single training experience (Davis et al. 1989), and a similar temporal specificity as a function of training parameters has been replicated for different CRs in CFC (Bevins and Ayres 1995).The aim of Experiment 1 was to establish two different temporal predictions about US arrival, as a necessary step to test our hypotheses concerning the role of US temporal prediction error in memory destabilization-reconsolidation. Rats were trained with 1 and 5 min of context exposure prior to shock presentations, and tested $72 \mathrm{~h}$ later during $6 \mathrm{~min}$ in the training context, without shock. Freezing behavior was analyzed minute by minute.

Figure 1 depicts the experimental protocol (top panel) and the percentage of freezing behavior of both groups throughout the 6 min of reactivation (i.e., test) time (bottom panel). A mixed ANOVA on total freezing behavior during reactivation (training condition $\times$ time as factors) revealed no effect of training condition $\left(F_{(1,12)}=0.71, P=0.41\right)$, a significant effect of time $\left(F_{(5,60)}=10.16, P<0.05\right)$, and a significant interaction between both factors $\left(F_{(5,60)}=11.58, P<0.05\right)$. Thus, this result suggests that both learning conditions resulted in similar amounts of conditioned responding (i.e., memories of similar strength-see inset), although differentially distributed in time during the test session. Post hoc analyses revealed that groups did not differ between them during the first, third, or sixth minute of reactivation ( $P>0.98$ in all cases), but did so during the second, fourth, and fifth $(P<0.01$ in all cases). Further post hoc analysis for the 1-min learning condition revealed that $\mathrm{CR}$ in the second minute was higher than in the fourth and fifth minute $(P<0.001$ in all cases; Cohen's $f=0.87$ and Cohen's $f=1.22$ for comparison involving responding at the second versus fifth minute and second versus fourth minute, respectively) which correlates with the moment at which the US was delivered during training (within the first and second minute). Thereafter, CR decreased progressively. In the case of the 5-min condition, the post hoc analysis revealed that CR in the fifth minute was higher than in the first and second minute $(P<0.001$ in all cases; Cohen's $f=1.55$ and Cohen's $f=$ 1.08 for comparison involving responding at the second versus fifth minute and first versus fifth minute, respectively). In other words, a different pattern emerged with the 5-min condition: the CR progressively increased over time, reaching its maximum level within the fifth minute before falling sharply in the sixth minute, which correlates with US arrival during training. Such a distinctive variation of CR curves along testing time strongly suggests differential shock expectancies or predictions, determined by learning conditions. Moreover, in a nonreactivation control experiment (see Supplemental Fig. S1) these temporal 


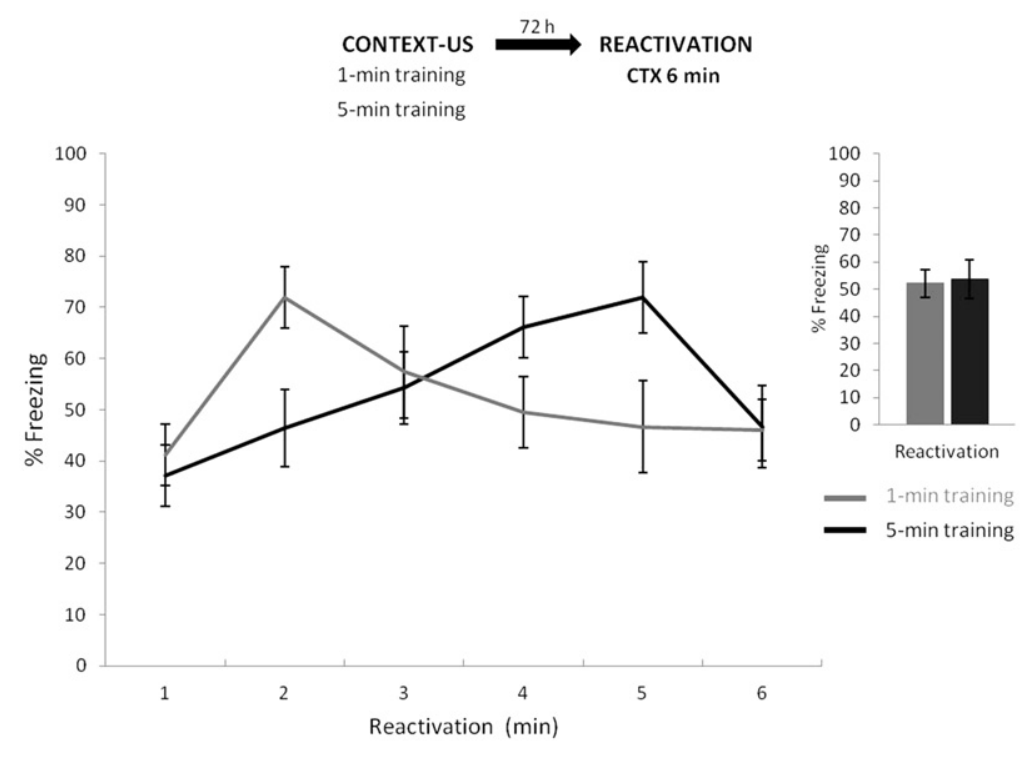

Figure 1. Experiment 1. Different temporal expectations about the US arrival as a function of the initial training parameters: a minute-by-minute analysis of behavior during a nonreinforced reactivation $3 \mathrm{~d}$ later revealed precise temporal control of the conditioned response (CR, freezing), for it peaked and decreased according to the time point at which the US was delivered during training (1 and $5 \mathrm{~min}$ ). Inset shows freezing over the 6 min during test. Data are expressed as means \pm SEMs.

distributions of freezing were replicated, suggesting that the particular form of the CR in each single-event learning condition was not altered when MDZ or SAL were administered in the absence of memory reactivation.

\section{Experiment 2}

Experiment 1 suggests that the 1- and 5-min treatments lead to fear memories of similar strength but different temporal distributions. In order to confirm that a temporal prediction-error is critical to induce memory destabilization, two experiments were conducted in which shocks were administered during the reactivation session. In Experiment 2, rats trained in the 1- or 5-min conditions were retrained with identical parameters $72 \mathrm{~h}$ after the first training experience. In Experiment 3, shocks were presented 30 sec earlier than during original training, thus producing a discrepancy between expected and experienced shock arrival. Immediately after retraining, subjects were administered with MDZ or SAL. We hypothesized that under identical retraining conditions, MDZ would not lead to amnesic effects (Exp. 2), for the amnesic should only be effective when the retraining conditions are different from the previously stored temporal information (Exp. 3).

In addition to the above-mentioned objectives, we wanted to assess what effect, if any, does MDZ have on temporal control of behavior after post-training manipulations. Such analyses are available in the Supplemental Material under the heading Supplementary analysis (SA). A previous study used a design in which animals were retrained under similar or different temporal parameters and subsequently administered anisomycin in the lateral amygdala (Díaz-Mataix et al. 2013). Although they observed that the amnestic only exerted an effect when US timing was changed (by presenting shock before or after it was previously trained), they did not observe a precise temporal control. The present experiments investigated in CFC whether temporal control of behavior is preserved with the administration of MDZ after the induction of a US temporal prediction error.
The top panel of Figure 2 depicts the experimental protocol. Two groups of rats were trained (1- or 5-min conditions) and 3 d later retrained using identical parameters. Immediately after, half of the rats in each learning condition received a systemic injection of $3 \mathrm{mg} / \mathrm{kg} \mathrm{MDZ}$ (i.p.), while the other half received an equivalent amount of SAL. Twenty-four hours and $7 \mathrm{~d}$ later, rats were exposed to the training context without shock for 5 min (test and retest, respectively).

Figure $2 \mathrm{~A}$ shows the data for the 1-min condition, during retraining, test and retest. $T$-test analysis for the period of context exposure prior to shock during the retraining phase revealed no difference between groups $t_{(14)}=0.09, P=$ 0.92. A mixed ANOVA on test and retest data (drug $\times$ phase as factors) revealed no effect of drug $\left(F_{(1,14)}=0.36, P=\right.$ $0.55)$, phase $\left(F_{(1,14)}=0.48, P=0.49\right)$, or interaction between these factors $\left(F_{(1,14)}=0.12, P=0.73\right)$. Bayesian analysis found that the null hypothesis predicting no difference between SAL and MDZ groups in the test phase was 3.37 times more probable than the alternative hypothesis. Figure 2B shows the results for the 5-min condition during retraining, test, and retest, respectively. $T$-test analysis for the period of context exposure prior to shock during the retraining phase revealed no difference between groups $t_{(14)}=0.27, P=0.78$. A mixed ANOVA on test and retest data (drug $\times$ phase as factors) revealed no effect of drug $\left(F_{(1,14)}=0.17, P>0.68\right)$, phase $\left(F_{(1,14)}=1.08, P=0.31\right)$, or interaction between both factors $\left(F_{(1,14)}=1.61, P=0.22\right)$. Again, Bayesian analysis found that the null hypothesis predicting no difference in the CR in the test phase according to the drug treatment was 3.62 times more probable than the alternative hypothesis. For analyses of temporal control of behavior of both CFC protocols during test, see Supplemental Material SA1. Similar results were observed for both memories (Fig. 2C,D): the maximum levels of CR expression correlates with the moment at which the US was delivered both during the initial training and retraining. This suggests that the particular control of CR of each learning condition was preserved after $\mathrm{MDZ}$ administration, provided there was no US temporal prediction error during the reinforced reactivation.

Thus, the results suggest that an identical retraining session did not trigger plasticity processes sensitive to MDZ, presumably because no change in the temporal predictions between the previously stored information and the current experience (during retraining) was detected. In addition to similar levels of CR during test of both memories, the analyses of CR curves suggest that neither the application of MDZ nor SAL alter the expression of the specific temporal profiles.

\section{Experiment 3}

Experiment 3 was similar to Experiment 2, except that during retraining the parameters were altered by presenting the USs 30 sec earlier, compared with initial training. Therefore, animals were removed from the chamber $30 \mathrm{sec}$ earlier compared with the total exposure time of each learning condition during training. All other parameters were identical to previous experiments. We hypothesized that changing the retraining parameters would 

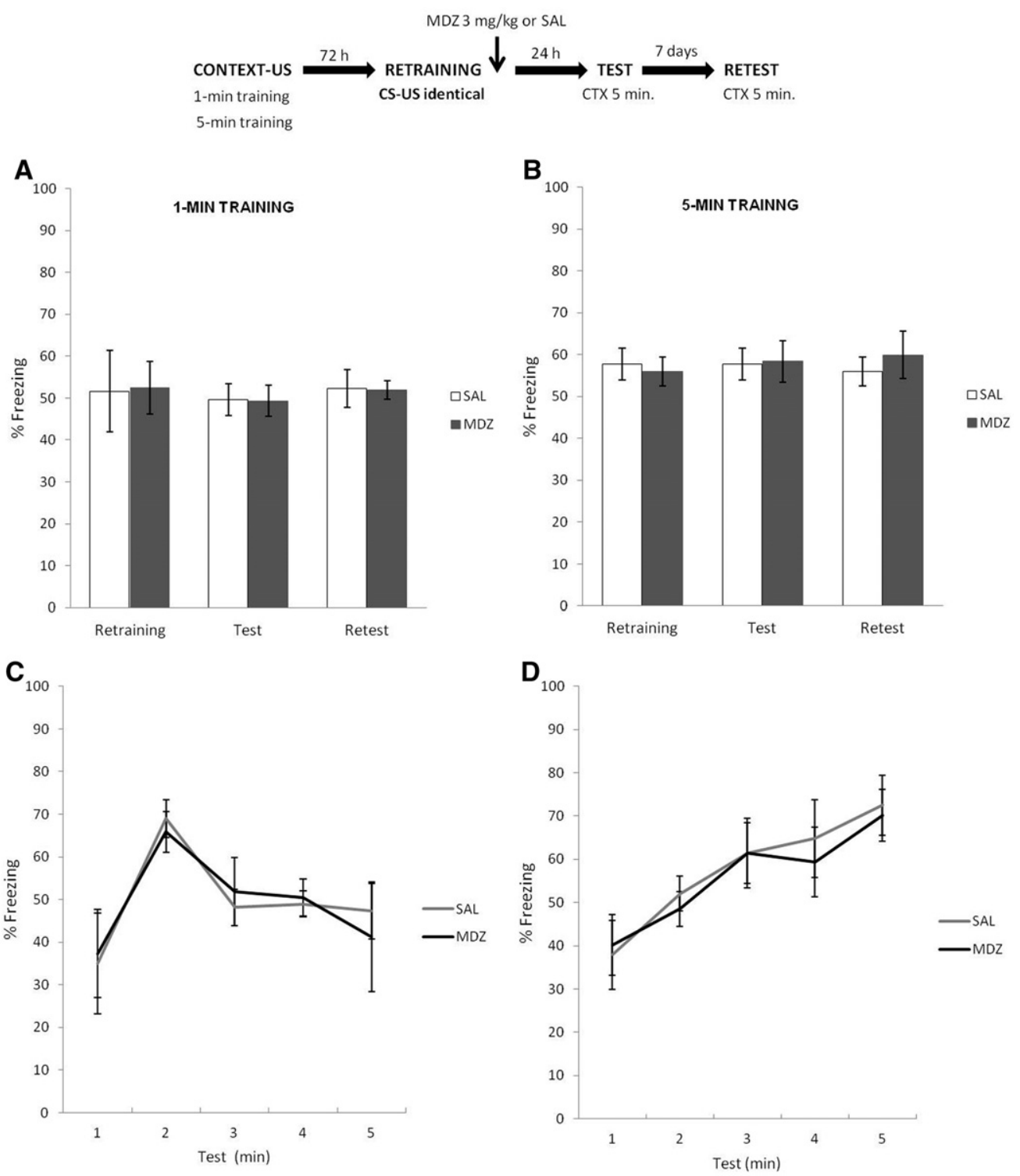

Figure 2. Experiment 2. In both learning conditions, MDZ administration did not have an amnestic effect when the retraining used exactly similar parameters as used during initial training. Furthermore, the control of CR of each learning condition was not altered by the amnestic. $(A, B)$ Both MDZ and SAL groups showed similar amounts of CR expression from test to retest, regardless of the initial training condition. ( $C, D$ ) In each learning condition, the $\mathrm{CR}$ increased and decreased $(1 \mathrm{~min})$ or increased $(5 \mathrm{~min})$ during test according to the time point at which the US was delivered during training. Data are expressed as means \pm SEMs.

lead to a US prediction-error and MDZ would exert its amnesic effects regardless of training condition. It was unclear however, whether MDZ would simply decrease conditioned responding without altering temporal control of behavior, or if temporal control would be lost with the administration of MDZ.

The top panel of Figure 3 depicts the experimental protocol. Figure $3 \mathrm{~A}$ shows the amount of freezing in the 1-min condition, during retraining, test, and retest. $T$-test analysis for the period of context exposure prior to shock during the retraining phase revealed no difference between MDZ and SAL groups: $t_{(12)}=0.16$, $P>0.87$. A mixed ANOVA on test and retest data (drug $\times$ phase as factors) revealed a main effect of drug $\left(F_{(1,14)}=174.49, P<\right.$ $0.01)$, no effect of phase $\left(F_{(1,14)}=0.43, P=0.83\right)$ and no interaction between these factors $\left(F_{(1,14)}=0.32, P=0.57\right)$. Post hoc analysis revealed that MDZ group expressed reduced freezing behavior in both test (Cohen's $f=3.07$ ) and retest (Cohen's $f=2.31$ ), relative to SAL group $(P<0.001$ in all cases). Figure 3B shows the amount of freezing in the 5-min condition during retraining, test and retest, respectively. A $t$-test analysis for the period of context exposure prior to shock during retraining phase revealed no difference between groups $t_{(14)}=0.75, P=0.47$. A mixed ANOVA on test and retest data (drug $\times$ phase as factors) revealed a main effect of drug $\left(F_{(1,14)}=164.20, P<0.01\right)$, an effect of phase $\left(F_{(1,14)}=5.79, P<0.05\right)$, and no interaction between these factors $\left(F_{(1,14)}=3.82, P=0.07\right)$. The post hoc analyses revealed that freezing behavior of MDZ group was significantly reduced during both test (Cohen's $f=2.46$ ) and retest (Cohen's $f=2.75$ ), when compared with SAL group $(P<0.001$ in all cases). A minute-by-minute analysis of both CFC treatments was used to assess whether a change in temporal parameters would reveal an effect of the 


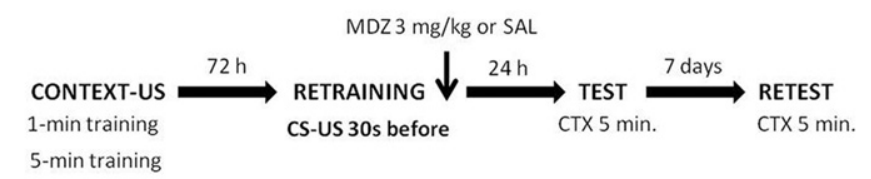

A

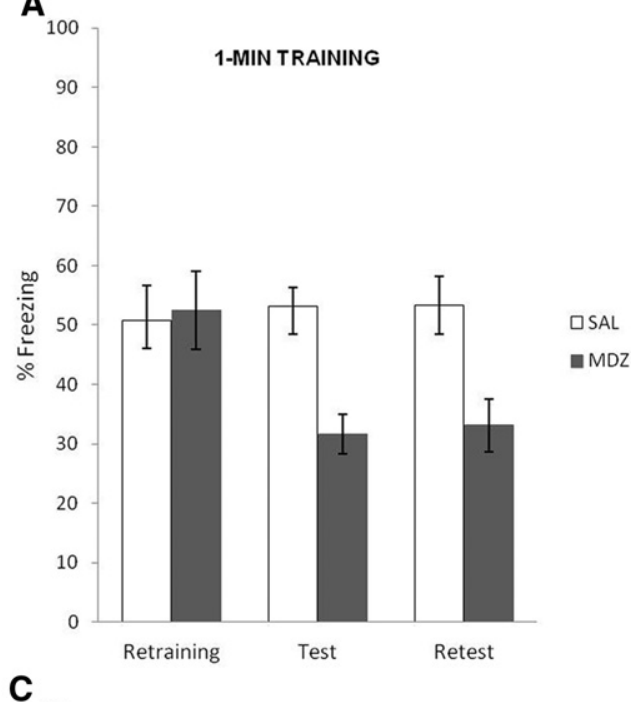

C

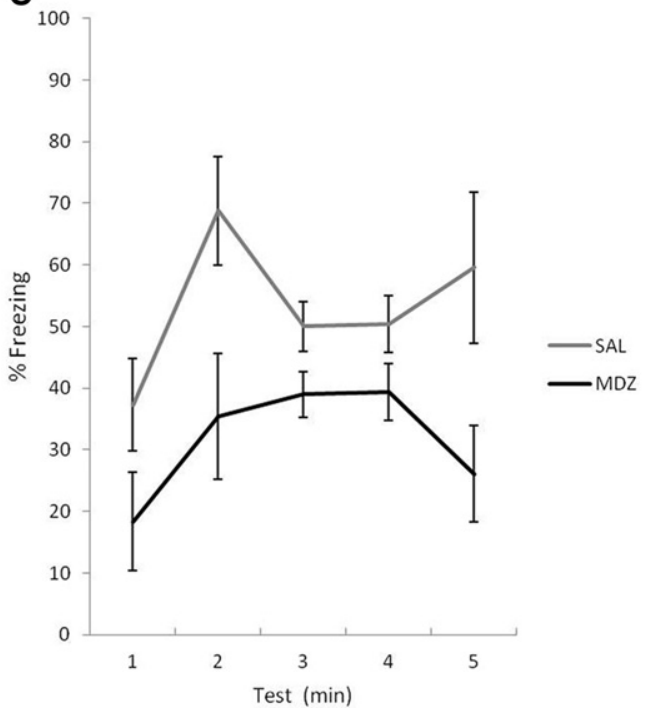

B

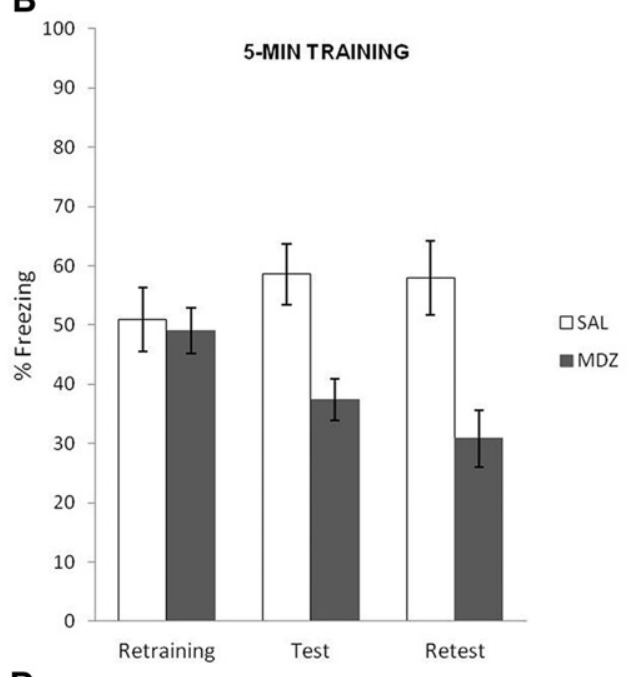

D

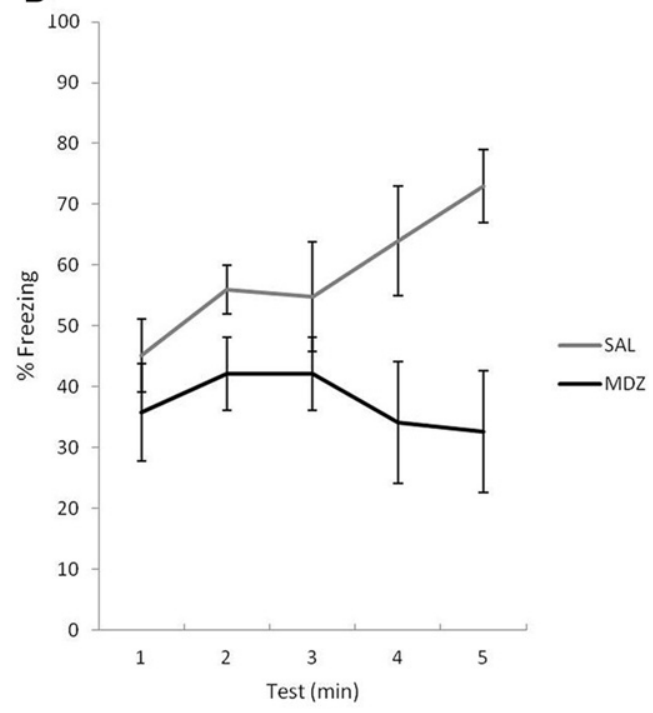

Figure 3. Experiment 3. When, throughout the reinforced reactivation the US was administered 30 sec before according to the original training parameters, MDZ administration had an amnesic effect. Theoretically, US temporal prediction error was detected during the reinforced reactivation, after which destabilization-reconsolidation was triggered. $(A, B)$ In both learning conditions, after MDZ administration, MDZ rats expressed significantly less CR expression than SAL group during both test and retest. $(C, D)$ While in each learning condition MDZ groups lost the specific temporal profiles, SAL groups revealed a clear temporal control of CR expression, for it peaked and decreased according to the time point at which the US was delivered during the initial training. Data are expressed as means \pm SEMs.

amnestic that is related or not to temporal control of behavior (see Supplemental Material SA2). Notably, for both learning conditions the analyses revealed temporal control of CR expression (SAL groups), for it peaked and decreased according to the time point at which the US was delivered during the initial training. MDZ not only decreased CR, but also altered the temporal control of behavior (Fig. 3C,D).

These results show that post-reactivation plasticity processes (i.e., memory destabilization-reconsolidation) can be induced when all the conditions presented during initial training are available, as long as there is a US prediction error based on stored tem- poral estimations and current (new) experience. Hence, these results strengthened the hypothesis that US temporal error detection is critical to trigger memory destabilization, rather than the mere reactivation of the already consolidated memory; and they are also consistent with a recent report by Díaz-Mataix et al. (2013). However, in that report, temporal control of behavior was not precise and thus it is unclear whether the amnestic altered memory strength or temporal control. The present results suggest that, in addition to lower CR, temporal control was lost only when there was a change in temporal parameters from training to retraining. 


\section{Experiment 4}

According to the prediction-error hypothesis, the processes of memory destabilization-reconsolidation will only take place if a discrepancy between stored information and current experience is detected during reactivation (Exp. 3). It has been reported by several laboratories (Eisenberg et al. 2003; Pedreira and Maldonado 2003; Suzuki et al. 2004; Bustos et al. 2009) that a short memory reactivation only produces memory retrieval (with or without CR expression). In a recent study (Piñeyro et al. 2013; Exp. 1) we also observed in this preparation that a short reactivation did not result in memory destabilization Therefore, we predicted that a 30-sec reactivation session (without shock) in the conditioned context would not allow animals to detect any discrepancy with the already encoded information about US arrival, since in both single-event training conditions the US arrived at later time points (60 sec for the 1-min condition, $300 \mathrm{sec}$ for the 5 -min condition). As the secondary aim, we wanted to assess whether a short reactivation alters the temporally specific pattern of CR we observed in Exp. 1.

The top panel of Figure 4 depicts the experimental protocol. Rats were trained under 1- or 5-min conditions (as in previous experiments) and $3 \mathrm{~d}$ later received a brief context reexposure of $30 \mathrm{sec}$. This was followed immediately by a systemic injection of $3 \mathrm{mg} / \mathrm{kg} \mathrm{MDZ}$ or SAL (i.p.). Twenty-four hours and $7 \mathrm{~d}$ later, rats were exposed to the training context for $5 \mathrm{~min}$ without shock (test and retest, respectively). Figure $4 \mathrm{~A}$ shows the results of the 1 -min condition during reactivation, test and retest. $T$-test analysis of the reactivation phase revealed no difference between MDZ and SAL groups: $t_{(14)}=0.35, P=0.72$. A mixed ANOVA on test and retest data (drug $\times$ phase as factors) revealed no effect of drug $\left(F_{(1,14)}=2.00, P=0.17\right)$, no effect of phase $\left(F_{(1,14)}=0.49\right.$, $P=0.49)$, and no interaction between these factors $\left(F_{(1,14)}=\right.$ $1.44, P=0.24)$. A Bayesian analysis found that the null hypothesis predicting no difference in the CR during the test phase according

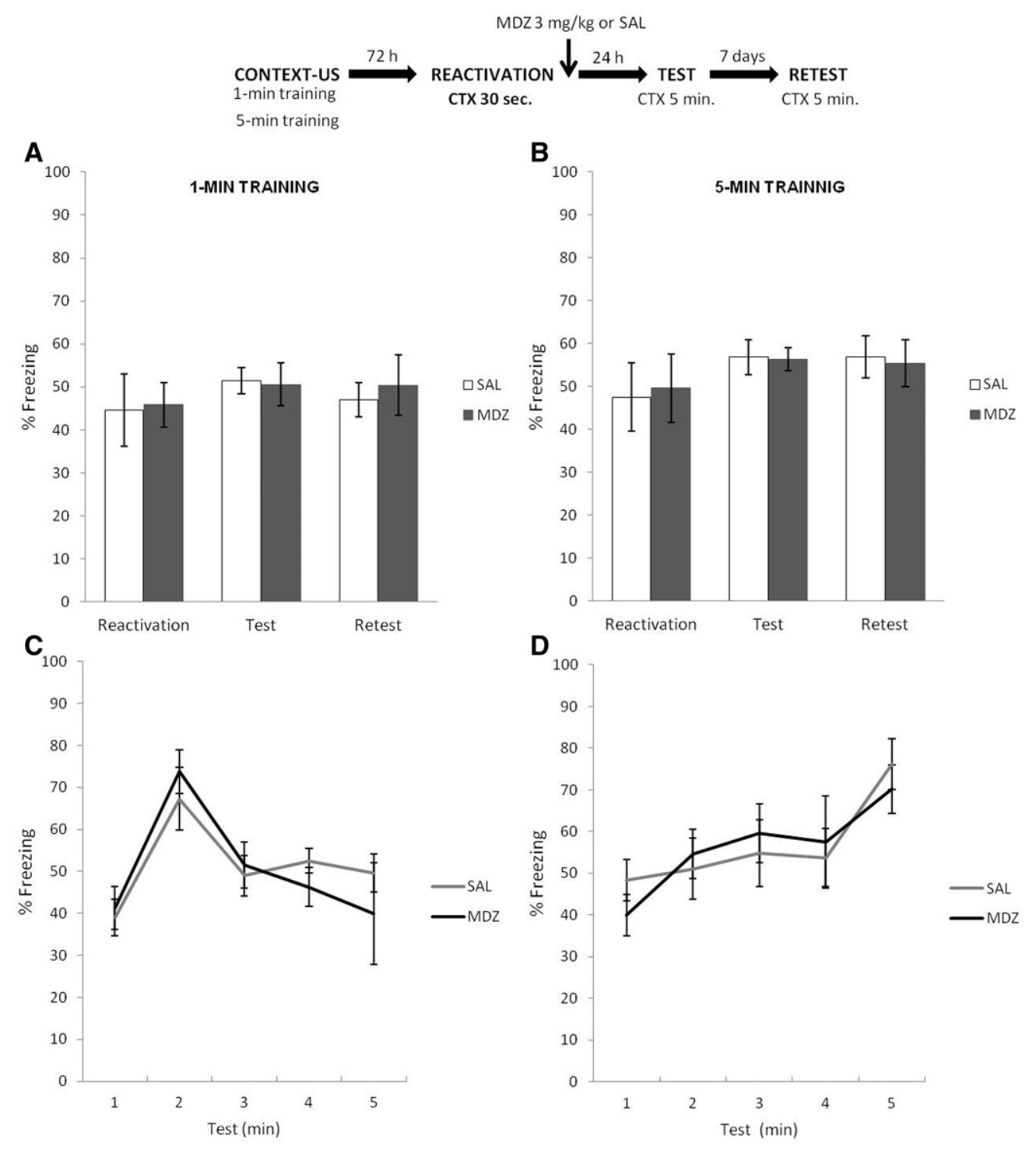

Figure 4. Experiment 4. When the reactivation context duration was less than that of training, the administration of MDZ did not have any amnesic effect, and further the amnestic agents did not alter the specific temporal control of CR. $(A, B)$ Both MDZ and SAL groups showed similar amounts of CR expression during test and retest, regardless of the initial training condition. $(C, D)$ the specific control of $C R$ form was not altered by MDZ. In each learning condition, the CR changes according to the time point at which the US was delivered during training. Data are expressed as means \pm SEMs. 
to the drug treatment was 3.62 times more probable than the alternative hypothesis. Figure $4 \mathrm{~B}$ shows the results for the 5-min condition during reactivation, test, and retest. $T$-test analysis of the reactivation phase revealed no difference between MDZ and SAL: $t_{(14)}=0.13, P=0.89$. A mixed ANOVA on test and retest data (drug $\times$ phase as factors) revealed no effect of drug $\left(F_{(1,14)}=\right.$ $0.84, P=0.37)$, phase $\left(F_{(1,14)}=1.35, P=0.26\right)$, or interaction between these factors $\left(F_{(1,14)}=0.79, P=0.38\right)$. Again, using a Bayesian analysis of the MDZ and SAL data in the test phase, we found that the null hypothesis was 3.70 times more probable than the alternative hypothesis, proposing a difference based on the drug treatment. We additionally analyzed temporal control of behavior during test using a minute-by-minute mixed ANOVA for each training condition (see Supplemental Material SA3). Notably, in both cases the CR peak correlates with the period of time at which the US was delivered in each learning condition (Fig. 4C,D). Thus, a short reactivation that does not result in memory destabilization, does not alter the temporal profile of the fear memories.

In conclusion, these results suggest that MDZ does not have amnesic effects on CFC memory when administered after a brief $(30 \mathrm{sec})$ reactivation, regardless of the initial training condition. In line with our reasoning, 30 sec of context reexposure was insufficient time to violate the predictions about the US arrival for either learning condition, and destabilization-reconsolidation processes were not observed.

\section{Experiment 5}

If the temporal prediction about US arrival is an attribute of the reactivated memory, we expected that 2 min of context reexposure (without shock) should be sufficient to destabilize the memory trained with 1-min of context exposure, but not the one trained with 5-min of context exposure. Thus, we predicted amnesic effects of MDZ only for animals trained in the 1-min condition. In addition, we wanted to assess whether memory destabilization induced by US temporal prediction error has an effect on the temporal distribution of CR at test.

The top panel of Figure 5 depicts the experimental protocol. It was similar to that used in Experiment 4, except that context reexposure (i.e., reactivation) lasted for $2 \mathrm{~min}$, instead of $30 \mathrm{sec}$. Figure $5 \mathrm{~A}$ shows the results for the 1-min condition during reactivation, test, and retest. $T$-test analysis for the reactivation phase revealed no difference between MDZ and SAL groups: $t_{(14)}=$ 1.04, $P=0.31$. A mixed ANOVA on test and retest data (drug $\times$ phase as factors) revealed a main effect of drug $\left(F_{(1,14)}=408.19\right.$, $P<0.01)$, an effect of phase $\left(F_{(1,14)}=18.07, P<0.01\right)$, but no interaction $\left(F_{(1,14)}=2.72, P=0.12\right)$. Post hoc analysis revealed that freezing behavior for MDZ group was significantly reduced during both test (Cohen's $f=3.99$ ) and retest (Cohen's $f=4.00$ ), relative to SAL group $(P<0.001$ in all cases). Figure $5 \mathrm{~B}$ shows the results for the 5-min condition during reactivation, test, and retest. $T$-test analysis for the reactivation phase revealed no difference between MDZ and SAL: $t_{(14)}=0.10, P>0.92$. A mixed ANOVA on test and retest data (drug $\times$ phase as factors) revealed no effect of drug $\left(F_{(1,14)}=0.04, P=0.82\right)$, phase $\left(F_{(1,14)}=0.23, P=0.63\right)$, or interaction between these factors $\left(F_{(1,14)}=0.63, P=0.44\right)$. A Bayesian analysis supported the null hypothesis, it was found to be 3.41 times more likely than the alternative.

In sum, Experiment 5 suggests that, after a 2-min context reactivation, MDZ only produced amnesic effects in animals trained with $1 \mathrm{~min}$ of context exposure, having no amnesic effect in animals trained under the 5-min condition. These conclusions are strengthened by the minute-by-minute analyses (see Supplemental Material SA4), which indicate that 2 min of nonreinforced context exposure for the 5-min condition does not alter the temporal profile of the CR (Fig. 5D), presumably because 2 min still does not allow the detection of a temporal prediction error about the US arrival, as observed in Experiment 4 for the 1 min condition in which the precise temporal controls of CR were unchanged after a mere retrieval. In contrast, a temporal predictive error seems to have occurred for 1-min rats, as inferred by the lack of temporal precision of the CR of both MDZ and SAL groups. Notably, the occurrence of an US temporal prediction error during the nonreinforced reactivation of context reveals an amnesic effect of MDZ which is also related to temporal control of behavior (Fig. 5C). This suggests that a 2-min nonreinforced context reactivation leads to prediction error only for animals trained in the 1-min condition. Consequently, if temporal prediction error about US arrival is necessary for memory destabilization, extending the reactivation duration should induce memory destabilization-reconsolidation processes for animals trained in the 5-min condition. Testing this prediction was the aim of Experiment 6 .

\section{Experiment 6}

In line with the reasoning of Experiment 5, we hypothesized that 6 min of reactivation would be sufficient for the animals trained in the 5-min condition to detect a relevant difference relative to their initial learning (i.e., US absence at the expected point in time). Furthermore, we assessed whether 6 min of context reactivation would induce extinction learning in the animals trained under the 1-min condition. As mentioned in the Introduction, previous studies have found that, with intermediate amounts of exposure which are beyond the period sufficient for memory destabilization, but not long enough to induce extinction learning, amnestics have no effect (Flavell and Lee 2013; Merlo et al. 2014; Sevenster et al. 2014). Accordingly, our prediction was that, after 6 min reactivation, MDZ would block memory reconsolidation for animals in the 5 -min condition, but perhaps have no effect on animals in the 1-min condition.

The top panel of Figure 6 depicts the experimental protocol. The only difference with Experiments 4 and 5 was that reactivation lasted $6 \mathrm{~min}$. Figure 6A shows the results for the 1-min condition during reactivation, test, and retest. $T$-test analysis for the reactivation phase revealed no significant difference between MDZ and SAL groups: $t_{(12)}=2.01, P=0.059$. A mixed ANOVA on test and retest data (drug $\times$ phase as factors) revealed no effect of drug $\left(F_{(1,12)}=0.84, P=0.37\right)$, phase $\left(F_{(1,12)}=1.07, P=0.32\right)$ or interaction between these factors $\left(F_{(1,12)}=1.89, P=0.19\right)$. A Bayesian analysis found that the null hypothesis predicting no difference between vehicle and MDZ conditions during the test phase was 3.47 times more probable than the alternative. Figure $6 \mathrm{~B}$ shows the results for the 5-min training condition during reactivation, test, and retest. $T$-test analysis for the reactivation phase revealed a significant difference between the groups MDZ and SAL: $t_{(14)}=2.86, P<0.01$. Although significant, the difference between MDZ and SAL groups during reactivation was minimal (54\% versus $57 \%$ of freezing, respectively) and likely the result of random variation, for no difference should have arisen before any pharmacological treatment was administered. Accordingly, we decided to analyze the data as in previous experiments. A mixed ANOVA on test and retest data (drug $\times$ phase as factors) revealed a main effect of $\operatorname{drug}\left(F_{(1,14)}=438.09, P<0.01\right)$, and no effect of phase $\left(F_{(1,14)}=3.07, P=0.10\right)$ or interaction between these factors $\left(F_{(1,14)}=1.55, P=0.23\right)$. The post hoc analysis revealed that the MDZ group expressed significantly less freezing behavior throughout the test (Cohen's $f=4.15$ ) and retest (Cohen's $f=$ $4.11)$ phases ( $P>0.01$ in all cases). For analyses of temporal control of behavior of both CFC protocols during test, see Supplemental Material SA5. These analyses suggest that in the 


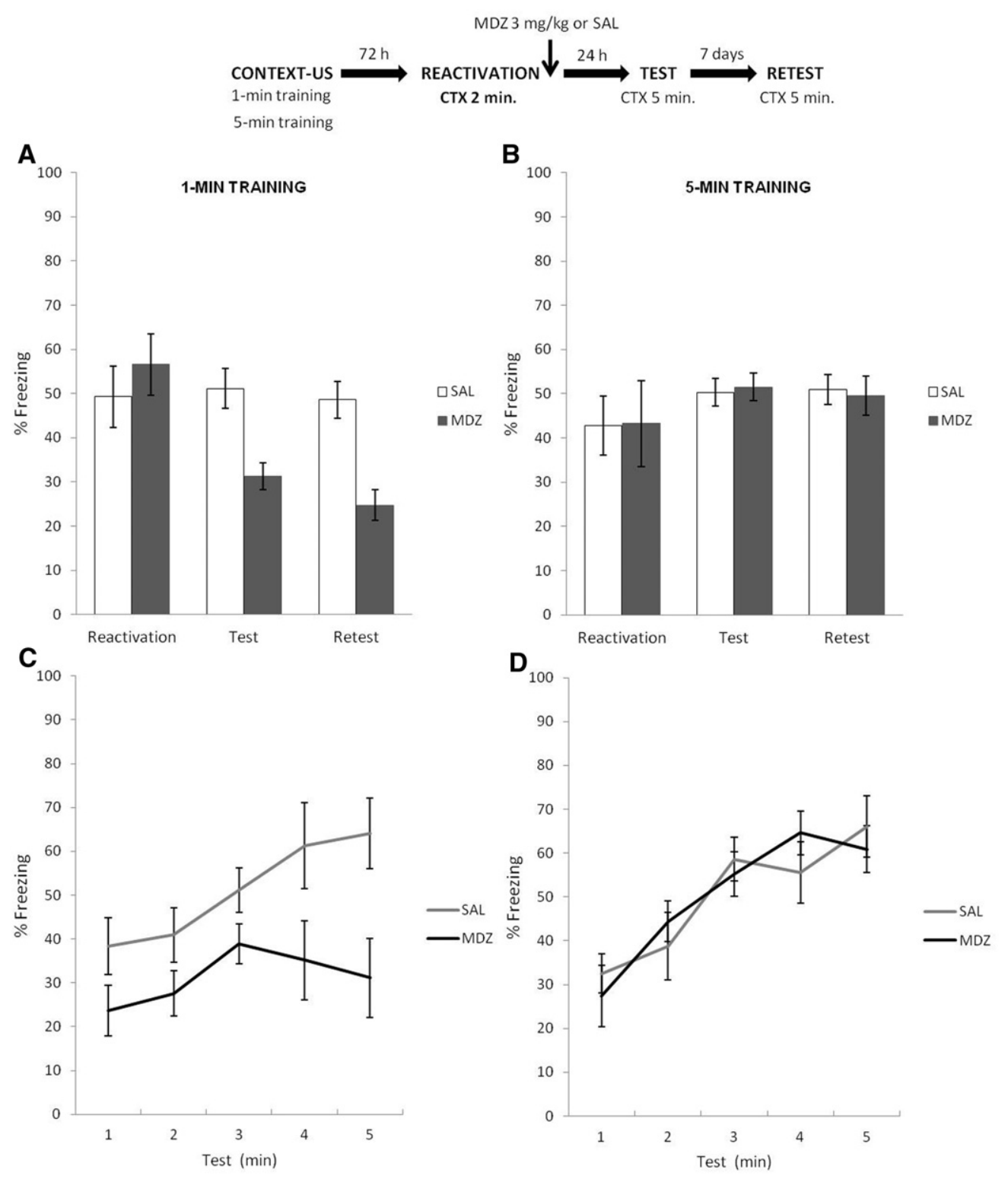

Figure 5. Experiment 5. Similar amounts of nonreinforced reactivation produce different memory outcomes, depending on the temporal expectations established during training. (A) In the 1-min condition, US temporal prediction error was detected during the nonreinforced reactivation after 2 min of reexposure, for MDZ group expressed significantly less CR expression than SAL group in both test and retest. (B) Two minutes of context reexposure was insufficient to violate the temporal expectation about the US arrival in the 5-min group. MDZ did not show amnesic effect in the CR expression neither test or retest. (C) the amnesic effect of MDZ was also related to temporal control of behavior. Both MDZ and SAL group showed a lack in the precise temporal control of the CR according to the initial training parameters. $(D)$ The CR temporal profiles were not altered by the application of amnestics after 2 min of nonreinforced reexposure. Data are expressed as means \pm SEMs.

1-min condition, the CR peaked and decreased according to the initial training parameters, whereas for the 5-min condition neither SAL nor MDZ groups showed the temporal control of the CR during test according to the previously established training parameters (Fig. 6C,D).

The aforementioned findings suggest two main conclusions: first, a 6-min reactivation session is sufficient to trigger destabilization-reconsolidation processes in animals trained in the 5 -min condition, as revealed by the amnesic effects of MDZ over the total percentage of CR expression and also in the temporally indistinct form of the CR distribution (Fig. 6D). Importantly, these results replicate the findings for the 1-min condition after 2 min of nonreinforced reactivation (Exp. 5; i.e., destabiliza- tion-reconsolidation). Overall, these results provide support for the critical role of US temporal prediction error in triggering destabilization-reconsolidation processes. Second, the same reactivation duration was neither long enough to generate extinction nor appropriate to induce destabilization-reconsolidation processes in animals trained in the 1-min condition. Consistent with findings from other laboratories (Flavell and Lee 2013; Merlo et al. 2014; Sevenster et al. 2014), these results suggest that there is a period of insensitivity to MDZ agent in which destabilization-reconsolidation processes are no longer taking place (deduced from the lack of amnesic effects of MDZ) and extinction learning has not yet been sufficiently developed. Thus, we suggest that this particular "null" outcome results from an interaction 


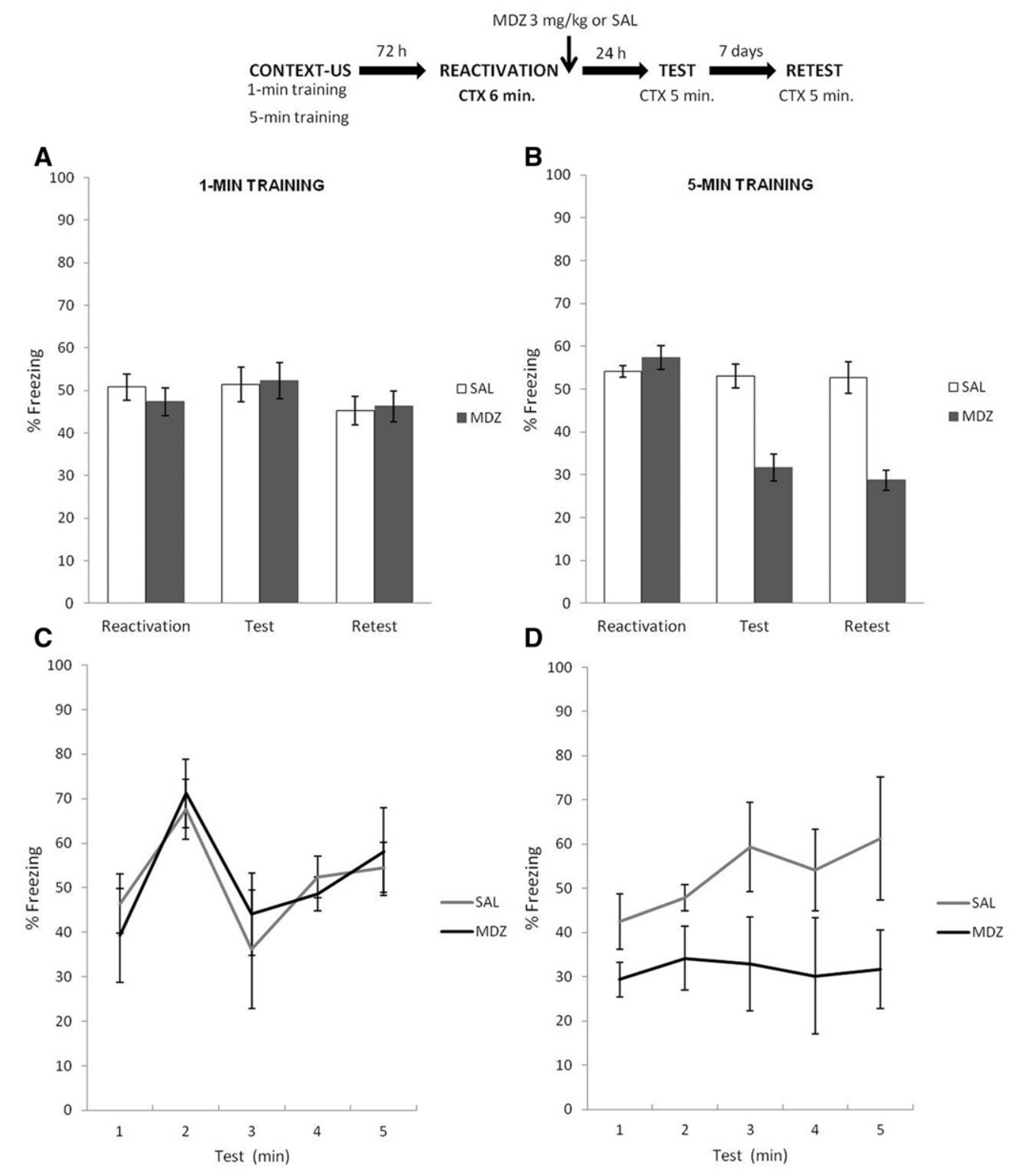

Figure 6. Experiment 6 . In the 5-min condition, MDZ administration after 6 min of nonreinforced reactivation session had an amnesic effect. However, as inferred from the lack of amnesic effect of MDZ, the 1-min condition revealed an insensitive period between destabilization-reconsolidation and extinction learning. (A) Administration of MDZ after 6 min of reactivation failed to reveal any amnesic effect. SAL group remained similar levels of CR from test to retest. Also (C) the minute-by-minute analysis suggested that both MDZ and SAL groups preserved the specific temporal control of CR. (B) Six minutes of nonreinforced reexposure was sufficient to violate the temporal prediction about the US arrival. Administration of MDZ revealed an amnesic effect; it expressed significantly less CR expression than SAL during both test and retest. In addition $(D)$ the amnesic effect was also related to the loss of the particular temporal control of the CR according to the initial training parameters. Data are expressed as means \pm SEMs.

between excitatory (CS-US) and inhibitory (CS-NoUS) memory traces, which, at this particular parametric point, are of equivalent strength and thus neither is sensitive to MDZ. Surprisingly, MDZ administered during this transitional insensitive period did not alter the temporal expectations about the US arrival that were encoded as a product of the specific training parameters (Fig. 6C).

\section{Experiment 7}

Previous experiments demonstrated that memory destabilization-reconsolidation processes can be modulated by different initial learning conditions and reactivation lengths (Exps 5 and 6). In other words, a reactivation procedure which destabilizes a memo- ry formed under certain training parameters may be ineffective when other parameters were used, because predictions about the US arrival were differentially encoded in each single-event training experience. These experiments also suggested the existence of a transition phase between destabilization-reconsolidation and extinction learning in which MDZ has no effect on memory expression. Since memory reactivation can also lead to extinction learning after extended CS exposure (Eisenberg et al. 2003; Briggs and Riccio 2007), it follows that when the CS-US and CS-NoUS traces are of similar strength they may cancel each other and result in neither memory trace being dominant-hence the amnestic is ineffective. In the next experiment, we attempted to answer two questions: first, what is the necessary time to trigger an extinction 
learning process for animals trained under the 1-min condition? Second, is there also a transitional insensitive period between destabilization-reconsolidation and extinction for the 5-min condition?

In Experiment 7, animals trained in the 1- and 5-min conditions were subsequently exposed to a 15 -min reactivation session, and followed by MDZ or SAL. Based on previous studies, we expected that $15 \mathrm{~min}$ should induce extinction learning (which would be blocked by MDZ) for animals trained under the 1-min condition (see Piñeyro et al. 2013). For animals trained in the 5-min condition, two possible outcomes were expected: the transitional insensitive period as observed in the 1-min condition (Experiment 6), particularly operationalized in the lack of MDZ amnesic effects and intact CR expression for SAL group, or extinction (reduced CR expression for SAL group, which would be blocked by MDZ).

The top panel of Figure 7 depicts the experimental protocol. Figure 7C shows the results for animals trained in the 1-min condition during reactivation, test and retest. $T$-test analysis for the reactivation phase revealed no difference between the MDZ and SAL groups, $t_{(14)}=0.68, P=0.63$. A mixed ANOVA on test and retest data (drug $\times$ phase as factors) yielded a main effect of drug $\left(F_{(1,14)}=128.06, P<0.01\right)$, a significant effect of phase $\left(F_{(1,14)}=\right.$ 72.81, $P<0.01$ ), and also a significant interaction between both factors $\left(F_{(1,14)}=114.64, P<0.01\right)$. Post hoc analyses revealed that groups differed from each other only during the test phase $(P<0.01$; Cohen's $f=4.12)$ : SAL group showed less CR expression when compared with MDZ group $24 \mathrm{~h}$ after the 15-min reactivation session, then this difference in the test between MDZ and SAL groups was lost $7 \mathrm{~d}$ later (retest; $P=0.99$ ). This was due to the SAL group expressing increased freezing from test to retest $(P<0.01$; Cohen's $f=3.71)$ suggesting spontaneous recovery, which is consistent with the typical pattern of extinction learning (Bouton 2004). The MDZ group, however, showed intact CR expression, a consequence of the amnesic agent over the consolidation of extinction memory, as previously reported with MDZ (Bustos et al. 2009) hypothermia (Briggs and Riccio 2007), and other pharmacological agents (Eiserberg et al. 2003; Pedreira and Maldonado 2003; Suzuki et al. 2004). Figure 7A shows the course of extinction minute by minute over the 15 min of reactivation time. For a minute-by-minute analysis of temporal control of behavior during reactivation and test sessions, see Supplemental Material SA6. During the test session neither SAL nor MDZ groups showed the temporal control of the CR according to the initial learning parameters (Fig. 7E).

Figure 7D shows the results for the 5 -min condition. $T$-test for the reactivation phase revealed no difference between the MDZ and SAL groups, $t_{(14)}=0.04, P>0.96$. A mixed ANOVA on test and retest data (drug $\times$ phase as factors) showed no effect of drug $\left(F_{(1,14)}=1.55, P=0.23\right)$, no effect of phase $\left(F_{(1,14)}=0.85\right.$, $P=0.36)$, and no interaction between these factors $\left(F_{(1,14)}=\right.$ $0.32, P=0.57)$. Bayesian analysis found that the null hypothesis predicting no difference between drug treatments in the test phase was 3.37 times more probable than the alternative hypothesis. Figure 7B shows minute by minute the 15 min of reactivation time. Temporal control of behavior was assessed during test and reactivation sessions using a minute-by-minute mixed ANOVA (see Supplemental Material SA6). In test session, the analysis showed that the temporal control of CR was unaltered in either group (Fig. 7F).

The behavioral pattern that emerges from this experiment is similar to the previous results, but using a slightly different set of parameters. In Experiment 6, we observed that $6 \mathrm{~min}$ of context reexposure for rats trained in the 1-min condition failed to reveal any effect of MDZ, and furthermore the analysis of the CR form suggested that both MDZ and SAL groups showed specific tempo- ral control of the CR. The current experiment replicated these results: 15 min of context reexposure for rats trained in the 5-min condition showed insensitivity to MDZ, it failed to induce extinction, and the minute-by-minute analysis also indicates that the particular temporal control of the CR was not altered.

\section{Experiment 8}

In Experiment 8, we wanted to determine whether extended context exposure would result in extinction learning for the 5-min condition. Only animals trained in the 5-min condition were used in this experiment, and we expected that extinction after 30 min of context exposure would be evident $1 \mathrm{~d}$ after training, but recover afterward (7 d later).

The top panel of Figure 8 depicts the experimental protocol. Rats trained with 5-min of context exposure prior to US arrival were subjected to $30 \mathrm{~min}$ of context reexposure and immediately after received MDZ or SAL (i.p.). Test and retest were identical to previous experiments. Figure $8 \mathrm{~B}$ shows the results during the different phases. $T$-test for the reactivation phase showed, as expected, no difference between groups: $t_{(12)}=1.44, P=0.17$. A mixed ANOVA on test and retest data (drug $\times$ phase as factors) revealed a main effect of drug $\left(F_{(1,12)}=53.54, P<0.01\right)$, a significant effect of phase $\left(F_{(1,12)}=22.38, P<0.01\right)$, and also a significant interaction effect between both factors $\left(F_{(1,12)}=11.20, P<0.05\right)$. Post hoc analyses revealed that the groups differed from each other during the test (SAL expressed significantly less freezing behavior than MDZ; $P<0.01$; Cohen's $f=4.58)$, but not during retest $(P=$ 0.97). At the same time, SAL group expressed increased freezing from test to retest $(P<0.01$; Cohen's $f=2.88)$ suggesting spontaneous recovery. Figure $8 \mathrm{~A}$ shows the course of extinction minute by minute over the $30 \mathrm{~min}$ of reactivation time. For a minute-by-minute analysis of temporal control of behavior during reactivation and test sessions, see Supplemental Material SA7. Critically, during the test session, the analyses suggested that the temporal specificity of CR form seems not to be strongly modified in the MDZ group (Fig. 8C). These results suggest that extinction learning can be achieved through a 30 min unreinforced reactivation for animals trained under the 5-min condition. This was evidenced by a reduced CR expression during test that recovered spontaneously a week later, an effect blocked by MDZ. It is worth noting that during long extinction sessions, neither learning condition (Figs 7A, B, 8A) showed a complete reduction in levels of CR. This is consistent with other studies using single-session extinction in rodents (Cain et al. 2003) and humans (Tsao and Craske 2000). It is unclear whether the reduction of fear during extinction predicts fear expressed during subsequent tests (for review, see Craske et al. 2014).

\section{Discussion}

The results in this study suggest a number of conclusions. First, as previously suggested, time is encoded as a component of associative memories and critically determines the timing of conditioned responding, possibly through temporal predictions about the reinforcer onset (Savastano and Miller 1998; Gallistel and Balsam 2014). In addition, US temporal prediction-error determines the extent to which reactivation destabilizes a memory trace thus making it vulnerable to the effects of an amnestic agent (Díaz-Mataix et al. 2013).

Second, whichever of four possible outcomes occurs after memory reactivation depends on specific training conditions. This conclusion was reached by a comprehensive combination of learning and reactivation conditions that shed light on the relationship between the initial learning conditions ( 1 or $5 \mathrm{~min}$ ) and the four different outcomes during the reactivation of 

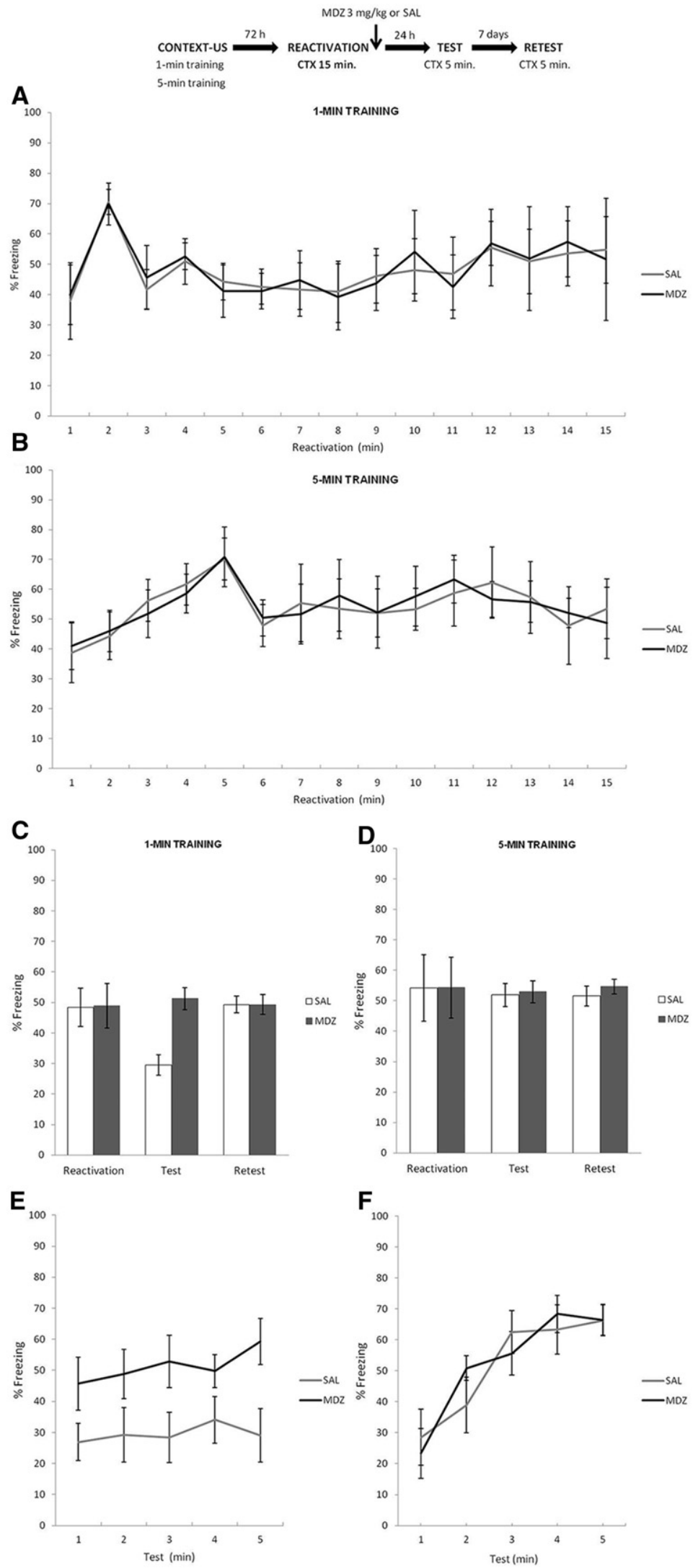

Figure 7. Experiment 7. In the 1-min condition, 15 min of nonreinforced reexposure were sufficient to result in long-term extinction memory, but a different mnemonic outcome emerged in the case of 5-min condition with similar nonreinforced reactivation time (i.e., transitional insensitive period). $(A, B)$ During reactivation, the CR of both learning conditions peaked and decreased according to the previously temporal parameters established in each training protocol. (C) MDZ administration increased responding when it was administered after a reactivation, which lasted 15 min, consequence of the amnesic agent interfering with extinction. SAL group expressed significantly less CR expression than MDZ in test, but the difference between both groups was lost $7 \mathrm{~d}$ later (i.e., spontaneous recovery). On the contrary, $(D)$ administration of MDZ after 15 min of nonreinforced CTX exposure for 5-min animals failed to reveal any amnesic effect over the CR expression during test and retest. Finally, $(E)$ while the particular control of conditioned response was lost after the occurrence of extinction learning, $(F)$ no changes in the particular temporal control of CR were produced by the administration of MDZ or SAL in the 5-min training condition. Data are expressed as means \pm SEMs. 


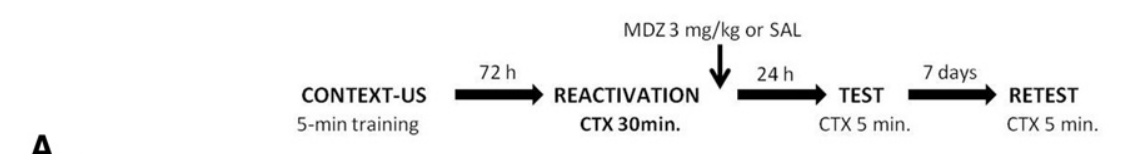

\section{A}
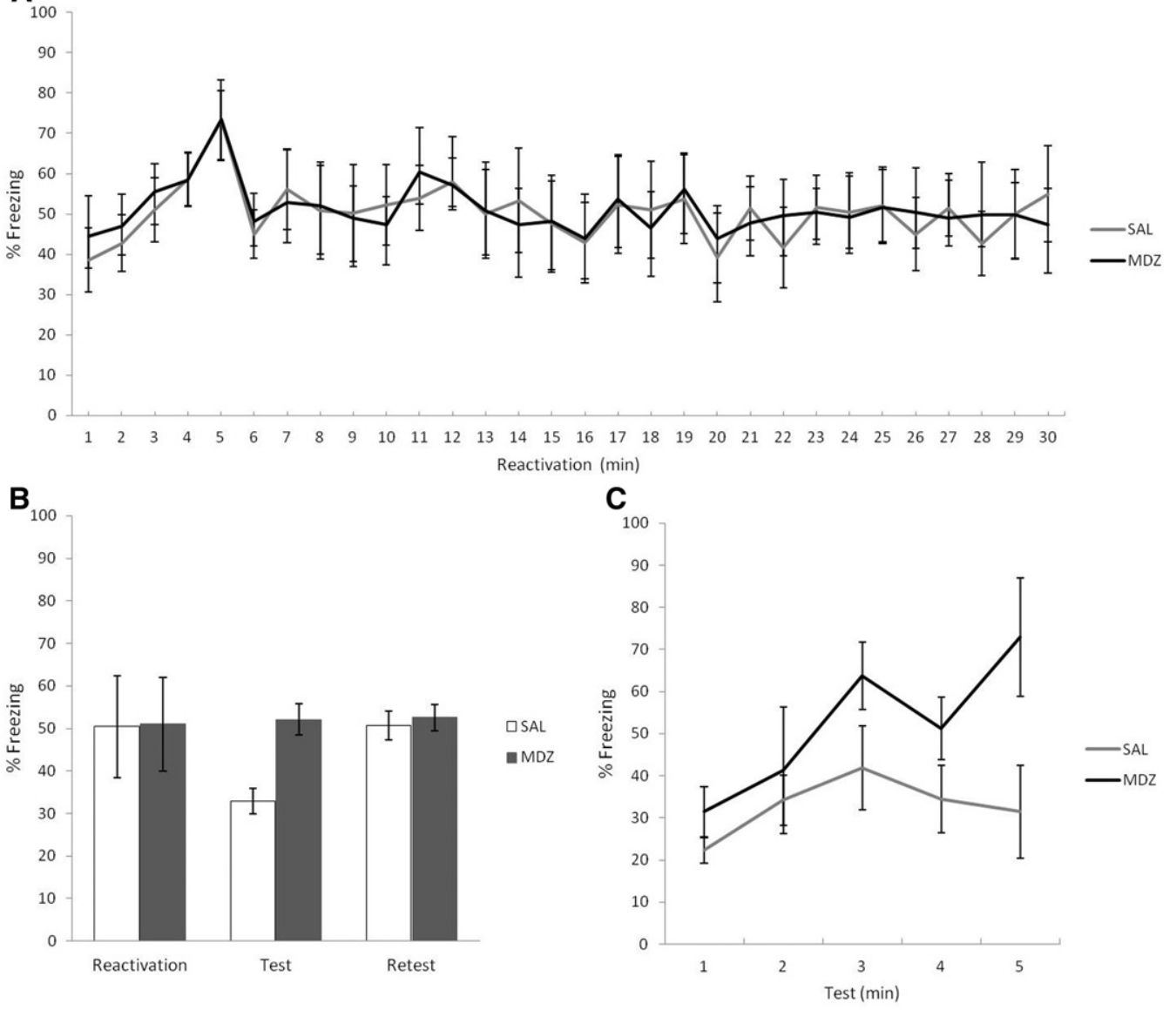

Figure 8. Experiment 8. Thirty minutes of nonreinforced CTX exposure were sufficient to result in a long-term extinction memory for 5-min condition. (A) During reactivation, the particular control of conditioned response is observed in the first minutes; it peaked and decreased according to the previously temporal parameters established in the training protocol. (B) MDZ administration interfered with extinction learning. SAL group significantly expressed less CR than MDZ during test, but this difference was lost $7 \mathrm{~d}$ later (retest) because SAL showed an increase on CR from test to retest (i.e., spontaneous recovery). (C) In the test SAL group lost the specific control of CR after 30 min of nonreinforced reexposure, and MDZ group showed a temporal control of CR expression, according to the initial training parameters. Data are expressed as means \pm SEMs.

previously encoded memories. We first observed that the induction of an US temporal prediction error can occur when the US was presented during memory reactivation, provided that the temporal parameters of training were altered during retraining (Díaz-Mataix et al. 2013). This demonstrated that a difference between what is expected and what is experienced (i.e., US temporal prediction error) is critical for memory destabilization and subsequent reconsolidation. This suggestion was strengthened by the fact that the induction of an US temporal prediction error does not only occur in the presence of the US. During nonreinforced reactivation, memory destabilization (and subsequent vulnerability to MDZ) was only observed when subjects were exposed to the trained context long enough for omission of the expected reinforcement to be experienced. The working hypothesis that unreinforced reactivation of such temporally specific memories may differentially result in mere retrieval, destabilization-reconsolidation, transitional insensitive period or extinction depending on the conditions of training and the amount of reactivation, was supported by the different reactivation lengths that trigger destabilization-reconsolidation processes for each CFC memory.
We also observed that during post-training CS exposure, less exposure than that used during training is sufficient for memory retrieval (i.e., expression of CR) but it does not lead to memory destabilization, as inferred from the lack of effect of MDZ upon subsequent memory expression (Exps 4 and 5). Finally, similar results emerged in the case of the transitional insensitive period and extinction learning. That is, different amounts of nonreinforced reactivation were needed to reveal these memory outcomes by each CFC protocol (Exps 6A, 7, and 8). We return to the implications of these later.

Consistent with the present results, a study has also suggested that US temporal prediction error triggers memory destabilization (Díaz-Mataix et al. 2013). In the present research we first replicated the findings of Díaz-Mataix et al. (2013; Exps 2 and $3)$, but then we studied prediction error after different amounts of nonreinforced reactivations, which demonstrated that the US temporal prediction error can induce memory destabilization in the absence of shock. In addition, the measurement of US expectancies operationalized by the temporal CR changes during nonreinforced reactivation could provide an independent measure 
of the memory reconsolidation process itself. This is in line with recent suggestions that the period of time of maximum US expectancy (i.e., fear) is a key factor to improve therapeutic strategies such as enhancing inhibitory learning based on an expectancy violation model (Craske et al. 2014). In addition, this is also in agreement with reports in humans (Sevenster et al. 2013, 2014) which strongly indicate that post-retrieval changes in US-expectancy can be used to reveal the different processes triggered by nonreinforced memory reactivation.

This study is first to demonstrate that when a US temporal prediction error is detected during nonreinforced reactivation, temporal-specific control of behavior is lost, as also does extinction learning (for a summary of temporal control of CR throughout Exps 2-8, see Supplemental Material Table S1). On the contrary, when US prediction error is not detected after insufficient reactivation time or when reactivation length is beyond reconsolidation (but not enough to establish an extinction memory) the temporal-specific control of CR is preserved, without altering the distribution of responding as a function of each particular single-event training parameter. However, it is important to note that additional minutes of context reexposure which are beyond the period sufficient for memory destabilization, but not long enough to induce extinction learning, restore temporal control that was lost after memory destabilization. This point should be addressed in future studies.

The present experiments were designed under the premise that multiple outcomes are possible after memory reactivation, and that US temporal prediction error can serve as indicative of when a memory will be most vulnerable to the effects of an amnestic. Simply put, when the training conditions are known, US temporal prediction error can dictate when the amnestic will be effective, following a relatively simple model such as that proposed by Sutton and Barto (1981). However, this model does not explain the lack of sensitivity to amnestic we observed with intermediate amounts of post-training CS exposure (Exps 6 and 7), because according to this model, there should still be some error in such circumstances. Neither can simple temporal prediction error account for the vulnerability of extinction memories to MDZ (Exps 7 and 8), because at the end of extinction prediction error should be minimal. For decades now, it has been suggested that extinction is best captured by new inhibitory learning, which is context dependent (Bouton 1993). Thus, a modification of Sutton and Barto's architecture that assumes both excitatory CS-US and inhibitory CS-NoUS links has some advantages (Pan et al. 2008). This model is within the realm of temporal difference (TD) architecture, but by assuming two separate memory traces with different decay functions, it can better approximate critical aspects of extinction learning such as spontaneous recovery. A similar two-trace approach, but focused on attention was initially developed by Pearce and Hall (1980).

It is not entirely clear, however, how this model handles the lack of sensitivity to amnestics observed under both training conditions following intermediate amounts of exposure (also see Exton-McGuinness et al. 2014a for a potential explanation in terms of attentional processes to the CS). This finding is consistent with a report by Flavell and Lee (2013), using an appetitive learning task and MK-801 as amnesic agent. Merlo et al (2014) also reported a similar pattern in an aversive preparation. We suggest that for these models to handle the insensitivity to amnestics, a weighting rule such as that proposed by Devenport (1998) could be used to determine which (if any) memory trace will be dominant and hence vulnerable to the effects of amnestics.

Overall, the present results can best be accommodated appealing to two processes, namely temporal prediction-error (Sutton and Barto 1981) and trace dominance (Devenport 1998; Eisenberg et al. 2003). Prediction-error seems to be critical for memory destabilization, as suggested by the present results during both reinforced and nonreinforced memory reactivations. During nonreinforced reactivations, memory destabilization (and subsequent vulnerability to the effects of the amnestic MDZ) was only observed when subjects were exposed on extinction until the reinforcer was expected and not experienced (Exps 5 and 6). In addition, a change in shock expectancy by presenting the shock sooner than expected also revealed memory vulnerability to the amnesic (Exp. 3). However, prediction error does not explain the amnestic effects observed when post-training exposure was increased, assuming error was minimal when extinction learning has taken place. Presumably, when parameters favor extinction learning (or in other words, the formation of a new competing trace), it is this (CS-NoUS) mnemonic trace which is vulnerable to the amnestic effects, suggesting that, when multiple memory traces compete, prediction-error itself is insufficient to explain memory vulnerability. Instead, memory trace dominance seems to be the principle governing memory vulnerability (Eisenberg et al. 2003).

In summary, if basic knowledge on learning and memory mechanisms is to be translated to clinical settings (Urcelay 2012), it is imperative to know in detail the conditions and parameters that determine the occurrence of different outcomes when a previously consolidated memory is reactivated. The results from these studies indicate that the initial learning experiences can determine the reactivation length needed to trigger each of the four possible mnemonic outcomes, and point at two potential processes, namely temporal prediction-error and trace dominance.

\section{Materials and Methods}

\section{Subjects}

Subjects were experimentally naïve, adult male Wistar rats (60-65 $\mathrm{d}$ old, weighing 270-320 g at the beginning of the experiments). The animals were bred in our colony in the Laboratorio de Psicología Experimental, Facultad de Psicología, Universidad Nacional de Córdoba, Argentina. All animals were housed in standard laboratory Plexiglas cages $(60 \mathrm{~cm}$ long $\times 40 \mathrm{~cm}$ wide $\times 20 \mathrm{~cm}$ high) in groups of 3-4 per cage. Food and water were available ad libitum. Animals were maintained on a 12-h light-dark cycle (lights on at $8 \mathrm{a} . \mathrm{m}$ ), at room temperature of $21^{\circ} \mathrm{C}-23^{\circ} \mathrm{C}$. The standards of the NIH Guide for the Care and Use of Laboratory Animals were respected. The number of animals and their suffering was kept to the minimum possible to achieve the goals of this research.

\section{Drugs}

Midazolam (MDZ, Gobbi Novag SA, Buenos Aires, Argentina) was diluted in sterile isotonic saline (SAL, $0.9 \% \mathrm{w} / \mathrm{v}$ ) to a concentration of $3 \mathrm{mg} / \mathrm{mL}$, and administered intraperitoneally (i.p.). The total volume of drug or equivalent amount of SAL was $1.0 \mathrm{~mL} /$ $\mathrm{kg}$ in all cases. This dose of MDZ was selected on the basis of previous reports demonstrating its ability to block contextual fear memory reconsolidation in Wistar rats (Bustos et al. 2006, 2009).

\section{Apparatus}

Contextual Fear Conditioning was conducted in a 24 long $\times 22$ wide $\times 22 \mathrm{~cm}$ high Plexiglas chamber with opaque gray walls and a removable transparent ceiling, the floor consisting of 20 parallel stainless steel grid bars, each measuring $3 \mathrm{~mm}$ in diameter, spaced $1 \mathrm{~cm}$ apart and connected to a device to provide adjustable footshocks (Automatic Reflex Conditioner 7501, Ugo Basile, Milan, Italy). The chamber was cleaned with water and dried with paper towels before and after each experimental session. Recording of behavior (for off-line analysis) was made with a DCR-SR21 Sony Handycam digital video camera placed $50 \mathrm{~cm}$ 
above the conditioning chamber. Background noise was supplied with ventilation fans. All procedures occurred in a sound-attenuated experimental room. Experiments were always performed during the light phase of the diurnal cycle.

\section{Behavioral procedures}

In all experiments, rats were first identified, weighed and handled for $5 \mathrm{~min}$ on two separate days to habituate them to being handled. In those experiments involving i.p. injections, rats were also injected with $1 \mathrm{~mL} / \mathrm{kg}$ SAL after preexperimental handling was complete to habituate them to this procedure.

\section{Contextual fear conditioning}

One day after habituation, rats were taken individually from their home cage, transported into the experimental room and exposed to the conditioning chamber. Depending on the experiment: Athe animals were exposed for 1 min (group: "1-min") after which two footshocks $(1.0 \mathrm{~mA}, 3 \mathrm{sec}$ duration, with an intershock interval of $30 \mathrm{sec}$ ) serving as US were delivered. B-The animals were exposed for $5 \mathrm{~min}$ (group: 5-min), after which two footshocks $(1.0 \mathrm{~mA}, 3 \mathrm{sec}$ duration, with an intershock interval of $30 \mathrm{sec})$ serving as US were delivered. In both groups, immediately after the second shock ended, rats were removed from the chamber, transported back to the colony room and placed back in their home cages.

\section{Retraining session}

Seventy-two hours after conditioning, 1- and 5-min rats were fear conditioned again. Depending on the experiment, Experiment 2-The groups were retrained using similar parameters as that used in the initial training relative to context preexposure time ( 1 or $5 \mathrm{~min})$, total exposure time to CS-context (1 min $36 \mathrm{sec}$, and $5 \mathrm{~min} 36 \mathrm{sec}$ ), number (two footshocks), duration ( $3 \mathrm{sec}$ ), intensity $(1.0 \mathrm{~mA})$, and intershock interval $(30 \mathrm{sec})$ between the US (group "Ret CS-US identical"). Experiment 3-the groups were retrained using the same number, duration, intensity, and intershock interval between the USs, however both the context preexposure time and the total exposure time to CS were modified. For each learning condition the USs onsets were given "30 sec before," relative to the initial training parameters (group: "Ret CS-US 30 sec before). That is, for the 1-min condition the first US was given after $30 \mathrm{sec}$ of context exposure (instead of $60 \mathrm{sec}$ ); for the 5-min condition the first US was given after $4 \mathrm{~min}$ and $30 \mathrm{sec}$ (instead of $5 \mathrm{~min}$ ). The animals were removed from the chamber immediately after the second US. Thus, the animals were removed $30 \mathrm{sec}$ earlier than the total exposure time of each learning condition during training.

\section{Reactivation sessions}

Reactivations were always carried out $72 \mathrm{~h}$ after conditioning. Rats were reexposed to the conditioning chamber (i.e., context) without footshock, for different periods of time $(0.5,2,6,15$, and 30 min only for the group 5-min condition), depending on the experiment.

\section{Drug administration}

MDZ $3 \mathrm{mg} / \mathrm{kg}$ or an equivalent amount of SAL was injected i.p. immediately after reactivation sessions.

\section{Test}

Testing consisted of a 5-min exposure session to the conditioning chamber, without shock.

\section{Retest}

Retest was identical to test, but $7 \mathrm{~d}$ later (context-NoUS).

\section{Behavioral scoring}

All experiments were video-taped for later off-line analyses. Freezing behavior, defined as the total absence of body and head movements except for that associated with breathing (Blanchard and Blanchard 1969), was scored minute-by-minute with a stop-watch by an observer blind to the experimental condition of each animal, and expressed as \% of time (in seconds). Inter-observed reliability was established with a different set of data (Pearson's $r=0.95$ ).

\section{Experiments}

Due to the fact that throughout the experiments both learning conditions (1- and 5-min rats) were reactivated under similar amounts of time $(0.5,2,6$, and $15 \mathrm{~min})$, the experiments are presented in pairs, according to the reactivation context duration. However, with exception of Experiment 1, which included both conditions thereby providing some justification for the subsequent between-experiment comparisons, 1- and 5-min conditions were run separately. To avoid confounds that can arise from cross-experiment comparisons, the corresponding statistical analyses were performed separately within each experiment. This sort of presentation facilitates observing how different learning conditions produce different consequences for memory after similar amounts of reactivation.

\section{Experiment 1}

Two groups of rats were first randomly subjected to different training conditions, 1- or 5-min period. Seventy-two hours later, both groups were submitted to context reexposure of $6 \mathrm{~min}$. Group sizes were as follows: 1 - $\min (n=7)$ and 5-min $(n=7)$.

\section{Experiment 2}

(A) Seventy-two hours after conditioning, rats trained in the 1 -min condition were retrained under similar parameters as in the initial learning. Immediately after retraining, half of the rats received a $3 \mathrm{mg} / \mathrm{kg}$ MDZ injection (i.p) and the other half received an equivalent amount of SAL, then they were transported back to their home cages. Twenty-four hours and one wk after the injections, all groups were subjected to 5-min test. Group sizes were as follows: $\operatorname{MDZ}(n=8)$ and SAL $(n=8)$.

(B). Identical to the general procedures of Experiment 2A, except that the animals trained in the 5-min condition were retrained under similar conditions as used in the initial training. Group sizes were as follows: $\operatorname{MDZ}(n=8)$ and SAL $(n=8)$.

\section{Experiment 3}

(A) Identical to the general procedures of Experiment 2, except that the animals trained in the 1-min condition were submitted to a retraining where the first US arrived $30 \mathrm{sec}$ before, relative to its initial training condition. The other parameters (number, duration, intensity, and intershock interval between US) were not modified. Group sizes were as follows: $\mathrm{MDZ}(n=8)$ and SAL $(n=8)$.

(B) Identical to Experiment 3A, except that animals trained in the 5-min condition were submitted to a retraining where the first US arrived 30 sec before, according to the parameters of the learning condition. All other parameters remained intact, as in Experiment 3A. Group sizes were as follows: $\operatorname{MDZ}(n=8)$ and SAL $(n=8)$.

\section{Experiment 4}

(A). Rats were first subjected to a contextual fear conditioning with $1 \mathrm{~min}$ of exposure to the context. Seventy-two hours later, rats were subjected to $30 \mathrm{sec}$ of context reexposure, then half of the rats received a $3 \mathrm{mg} / \mathrm{kg}$ MDZ injection (i.p) and the other half receives an equivalent amount of SAL. Rats were labeled according to drug (MDZ or SAL). Twenty-four hours after the injections, all groups were subjected to 5-min test. One week later, all 
the groups were again subjected to 5 -min retest. Group sizes were as follows: $\operatorname{MDZ}(n=8)$ and SAL $(n=8)$.

(B) Identical to Experiment $4 \mathrm{~A}$, except that training condition was 5-min. Group sizes were as follows: $\operatorname{MDZ}(n=8)$ and SAL $(n=8)$.

\section{Experiment 5}

(A). identical to Experiment 4, except that the animals trained in the 1-min condition were submitted to $2 \mathrm{~min}$ of context reexposure, instead of $30 \mathrm{sec}$. Group sizes were as follows: $\mathrm{MDZ}(n=9)$ and SAL $(n=9)$.

(B). The only difference with the procedure of Experiment $5 \mathrm{~A}$ was the learning condition: the animals were trained in the 5 -min condition. Group sizes were as follows: $\operatorname{MDZ}(n=9)$ and $\operatorname{SAL}(n=9)$.

\section{Experiment 6}

(A). Identical to the general procedures of Experiments 4 and 5, except that the animal trained in the 1 -min condition were submitted to 6 min of context reexposure. Group sizes were as follows: $\operatorname{MDZ}(n=7)$ and SAL $(n=7)$.

(B). Identical to Experiment 6A, except that the animals were trained in the 5-min condition. Group sizes were as follows: MDZ $(n=7)$ and SAL $(n=7)$.

\section{Experiment 7}

(A). Identical to the general procedures of Experiments 4-6 except that the animals trained in the 1 -min condition were submitted to 15 min of context reexposure. $\operatorname{MDZ}(n=7)$ and SAL $(n=7)$.

(B). Identical to Experiment 7A, except that the animals were trained in the 5-min condition. Group sizes were as follows: MDZ $(n=8)$ and SAL $(n=8)$.

\section{Experiment 8}

Identical to the general procedures of Experiment 7, except that the animals trained in the 5-min condition were submitted to 30 min of context reexposure. Group sizes were as follows: MDZ $(n=8)$ and SAL $(n=8)$. The animals trained in the 1-min learning condition were not run with this reactivation time, because they reached the typical extinction parameters in Experiment 7A, with 15 min of context reexposure.

\section{Statistical analyses}

Results were expressed as mean \pm SEM of the percentage time the animal spent freezing. Data were analyzed with independent sample " $t$ " tests or mixed ANOVAS. The latter was followed with Newman-Keuls tests for post hoc analyses. Minute-by-minute mixed ANOVAs were used to assess temporal control of behavior in the first experiment, and it was followed by the NewmanKeuls test. In the case of the 1-min condition, the post hoc analyses compared the second minute with the fourth and fifth minutes during the test. For the 5-min condition, the fifth minute was compared with the first and second minutes. Cohen's $f$ was reported to indicate effect size based on specific one-way ANOVAs comparison when there were either a significant whiting-subject effect (between second versus fourth minute and second versus fifth minute for the 1-min learning condition, and between fifth versus second minute, fifth versus first for the 5-min learning condition) or a between-subjects effect in test or retest phases (between MDZ and SAL group). Effect sizes were classified as small $(f=0.2)$, medium $(f=0.5)$, and large $(\geq 0.8)$ according to Cohen (1992). In all cases, $P<0.05$ was the statistical threshold. The statistical package software STATISTICA 10 was used to analyze the data.

In several cases a manipulation, such as drug treatment, was critically found to have no effect on freezing behavior in the test phase. In these cases, we computed Bayes factors for use in supporting the null hypothesis (Rouder et al. 2009), using a freely available Bayes factor calculator (http://pcl.missouri.edu/ bayesfactor). In all cases, the scale of $r$ was set up to 1.0, which serves as a natural benchmark (Rouder et al. 2009).

\section{Acknowledgments}

This work was supported by a grant from Secretaría de Ciencia y Técnica (SeCyT) from the Universidad Nacional de Córdoba, Argentina, to A.M.B. J.M.A was supported by a pregraduate SeCyT studentship. R.I.F.M. was supported by a doctoral SeCyT studentship. We wish to thank to Ralph R. Miller for valuable comments on an earlier version of the manuscript.

\section{References}

Balsam PD, Drew MR, Yang C. 2001. Timing at the start of associative learning. Learn Motiv 33: 141-155.

Blanchard RJ, Blanchard DC. 1969. Passive and active reactions to fear-eliciting stimuli. J Comp Physiol Psychol 68: 129-135.

Bevins RA, Ayres JJB. 1995. One-trial context fear conditioning as a function of the interstimulus interval. Anim Learn Behav 23: 400-410.

Bouton ME. 1993. Context, time, and memory retrieval in the interference paradigms of Pavlovian learning. Psychol Bull 144: 80-99.

Bouton ME. 2004. Context and behavioral processes in extinction. Learn Mem 11: $485-494$.

Briggs JF, Riccio DC. 2007. Retrograde amnesia for extinction: similarities with amnesia for original acquisition memories. Learn Behav 35: $131-140$.

Bush RR, Mosteller F. 1955. Stochastic models for learning. Wiley, New York.

Bustos SG, Maldonado H, Molina VA. 2006. Midazolam disrupts fear memory reconsolidation. Neuroscience 139: 831-842.

Bustos SG, Maldonado H, Molina VA. 2009. Disruptive effect of midazolam on fear memory reconsolidation: decisive influence or reactivation time span and memory age. Neuropsychopharmacology 34: 446-457.

Cain CK, Blouin AM, Barad M. 2003. Temporally massed CS presentations generate more fear extinction than spaced presentations. J Exp Psychol Anim Behav Process 29: 323-333.

Cohen J. 1992. A power primer. Psychol Bull 112: 155-159.

Craske GM, Treanor M, Conway CC, Zbozinek T, Vervliet B. 2014. Maximizing exposure therapy: an inhibitory learning approach. Behav Res Ther 58: 10-23.

Davis M, Schlesinger LS, Sorenson CA. 1989. Temporal specificity of fear conditioning: effects of different conditioned stimulus-unconditioned stimulus intervals on the fear-potentiated startle effect. J Exp Psychol Anim Behav Process 15: 295-310.

Devenport LD. 1998. Spontaneous recovery without interference: why remembering is adaptative. Anim Learn Behav 26: 172-181.

Díaz-Mataix L, Ruiz Martinez R, Schafe G, LeDoux J, Doyére V. 2013. Detection of a temporal error triggers reconsolidation of amygdala-dependent memories. Curr Biol 23: 467-472.

Eisenberg M, Kobilo T, Berman DE, Dudai Y. 2003. Stability of retrieved memory: inverse correlation with trace dominance. Science 301: 1102-1104.

Exton-McGuinnes MTJ, Lee JLC. 2015. Reduction in responding for sucrose and cocaine reinforcement by disruption of memory reconsolidation. Eneuro 2: 1-17.

Exton-McGuinness MTJ, Lee JLC, Reichelt AC. 2014a. Updating memories - the role of prediction errors in memory reconsolidation. Behav Brain Res 278: 375-384.

Exton-McGuinness MTJ, Patton RC, Sacco LB, Lee JLC. 2014b. Reconsolidation of a well-learned instrumental memory. Learn Mem 21: $468-477$.

Flavell CR, Lee JLC. 2013. Reconsolidation and extinction of an appetitive Pavlovian memory. Neurobiol Learn Mem 104: 25-31.

Gallistel CR, Balsam PD. 2014. Time to rethink the neural mechanisms of learning and memory. Neurobiol Learn Mem 108: 136-144.

Guisquet-Verrier P, Riccio DC. 2012. Memory reactivation effects independent of reconsolidation. Learn Mem 19: 401-409.

Lee JLC, Milton AL, Everitt BJ. 2006. Reconsolidation and extinction of conditioned fear: inhibition and potentiation. J Neurosci 26: 10051-10056.

Lewis DJ. 1979. Psychobiology of active and inactive memory. Psychol Bull 86: $1054-1083$.

McGaugh JL. 1966. Time-dependent processes in memory storage. Science 153: $1351-1358$.

Merlo E, Milton AM, Goozée ZY, Theobald DE, Everitt BJ. 2014. Reconsolidation and extinction are dissociable and mutually exclusive processes: behavioral and molecular evidence. J Neurosci 34: 2422-2431.

Miller RR, Matzel LD. 2000. Memory involves far more than "consolidation." Nat Rev Neurosci 1: 214-216. 
Miller RR, Matzel LD. 2006. Retrieval failure vs. memory loss in experimental amnesia: definition and processes. Learn Mem 13: 491-497.

Misanin JR, Miller RR, Lewis DJ. 1968. Retrograde amnesia produced by electroconvulsive shock after reactivation of a consolidated memory trace. Science 160: $554-555$.

Nader K. 2013. The discovery of memory reconsolidation. In Memory reconsolidation (ed. Alberini P), pp. 1-9. Academic Press, UK.

Nader K, Schafe GE, LeDoux JE. 2000. Fear memories require protein synthesis in the amygdala for reconsolidation after retrieval. Nature 406: $722-726$.

Pan WX, Schmidt R, Wickens JR, Hyland B. 2008. Tripartite mechanism of extinction suggested by dopamine neuron activity and temporal difference model. J Nuerosci 28: 9619-9631.

Pavlov IP. 1927. Conditioned reflexes: an investigation of the physiological activity of the cerebral cortex. Oxford University Press, London.

Pearce JM, Hall G. 1980. A model for Pavlovian learning: variations in the effectiveness of conditioned but not of unconditioned stimuli. Psychol Rev 87: 532-552.

Pedreira ME, Maldonado H. 2003. Protein synthesis subserves reconsolidation or extinction depending on reminder duration. Neuron 38: $863-869$.

Pedreira ME, Pérez-Cuesta LM, Maldonado H. 2004. Mismatch between what is expected and what actually occurs triggers memory reconsolidation or extinction. Learn Mem 11: 579-585.

Piñeyro M, Ferrer Monti RI, Alfei JM, Bueno AM, Urcelay GP. 2013. Memory destabilization is critical for the success of the reactivation-extinction procedure. Learn Mem 21: 46-54.

Reichelt AC, Lee JLC. 2012. Appetitive Pavlovian goal-tracking memories reconsolidate only under specific conditions. Learn Mem 20: 51-60.

Reichelt AC, Exton-McGuinness MT, Lee JLC. 2013. Ventral tegmental dopamine dysregulation prevents appetitive memory destabilization. $J$ Neurosci 33: 14205-14210.
Rescorla RA, Wagner AR. 1972. A theory of Pavlovian conditioning: variations in the effectiveness of reinforcement and non-reinforcement. In Classical conditioning II: current research and theory (ed. Black AH, Prokasy WF), pp. 64-69. Appleton-Century-Crofts, New York.

Rouder JN, Speckman PL, Sun D, Morey RD, Iverson G. 2009. Bayesian t test for accepting and rejecting the null hypothesis. Psychon Bull Rev 16: $225-237$.

Savastano HI, Miller RR. 1998. Time as content in Pavlovian conditioning. Behav Processes 44: 147-162.

Sevenster D, Beckers T, Kindt M. 2012. Retrieval per se is not sufficient to trigger reconsolidation of human fear memory. Neurobiol Learn Mem 97: $338-345$.

Sevenster D, Beckers T, Kindt M. 2013. Prediction error governs pharmacologically induced amnesia for learned fear. Science 339: 830-833.

Sevenster D, Beckers T, Kindt M. 2014. Prediction error demarcates the transition from retrieval, to reconsolidation, to new learning. Learn Mem 21: 580-584.

Sutton RS, Barto AG. 1981. Toward a modern theory of adaptive networks: expectation and prediction. Psychol Rev 88: 135-170.

Suzuki A, Josselyn SA, Frankland PW, Masushige S, Silva AJ, Kida S. 2004. Memory reconsolidation and extinction have distinct temporal and biochemical signatures. J Neurosci 24: 4787-4795.

Tsao JCI, Craske MG. 2000. Timing of treatment and return of fear: effects of massed, uniform-, and expanding-spaced exposure schedules. Behav Ther 31: 479-497.

Urcelay G. 2012. Exposure therapy. In Exposure therapy: rethinking the model-refining the method (ed. Neudeck P, Wittchen H-U), pp. 35-63. Springer, New York.

Received March 3, 2015; accepted in revised form June 5, 2015. 


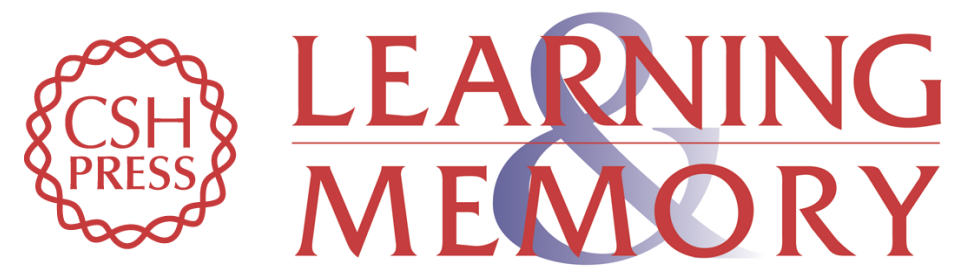

\section{Prediction error and trace dominance determine the fate of fear memories after post-training manipulations}

Joaquín M. Alfei, Roque I. Ferrer Monti, Victor A. Molina, et al.

Learn. Mem. 2015, 22:

Access the most recent version at doi:10.1101/Im.038513.115

\section{Supplemental http://learnmem.cshlp.org/content/suppl/2015/07/13/22.8.385.DC1 Material}

References This article cites 44 articles, 16 of which can be accessed free at: http://learnmem.cshlp.org/content/22/8/385.full.html\#ref-list-1

Creative This article is distributed exclusively by Cold Spring Harbor Laboratory Press for the Commons first 12 months after the full-issue publication date (see

License http://learnmem.cshlp.org/site/misc/terms.xhtml). After 12 months, it is available under a Creative Commons License (Attribution-NonCommercial 4.0 International), as described at http://creativecommons.org/licenses/by-nc/4.0/.

Email Alerting Receive free email alerts when new articles cite this article - sign up in the box at the Service top right corner of the article or click here. 